\title{
PROBLEMAS E PROBLEMATIZAÇÕES: IMPLICAÇÕES PARA O ENSINO DOS PROFISSIONAIS DA SAÚDE ${ }^{ \pm}$
}

\section{PROBLEMS AND PROBLEMATIZATIONS: IMPLICATIONS FOR THE EDUCATION OF HEALTH PRACTIONERS}

\author{
Wellington Barros da Silva ${ }^{1}$, Demétrio Delizoicov ${ }^{2}$ \\ ${ }^{1}$ Programa de Pós-graduação em Educação Científica e Tecnológica (PPGECT)/Universidade Federal de \\ Santa Catarina (UFSC), wbarrosdasilva@gmail.com \\ ${ }^{2}$ Programa de Pós-graduação em Educação Científica e Tecnológica (PPGECT)/Universidade Federal de \\ Santa Catarina (UFSC), demetrio@ced.ufsc.br
}

\section{Resumo}

O trabalho apresenta e discute, utilizando o referencial teórico de Paulo Freire os pressupostos epistemológicos e as características de duas estratégias de aprendizagem utilizadas no ensino superior na área da saúde: a Aprendizagem Baseada em Problemas (ABP) e a Metodologia da Problematização (MP). Estas metodologias fundamentam-se em diferentes visões teóricas e utilizam problemas como ponto de partida para o ensino e aprendizagem dos alunos. Na MP, os problemas são formulados pelos alunos a partir da observação da realidade. $\mathrm{Na} \mathrm{ABP}$, os problemas são elaborados por uma equipe de especialistas para contemplar conhecimentos e atingir objetivos de aprendizagem planejados. Com enfoques e pressupostos diferenciados quanto ao significado e objetivos do processo de problematização, as duas propostas trazem consigo implicações para a prática docente e para a formação dos profissionais da saúde.

Palavras-chave: Aprendizagem baseada em problemas, Problematização, Epistemologia, Educação superior em saúde.

\begin{abstract}
The article presents and argues, using the theoretical referential of educator Paulo Freire, the epistemological assumptions and the characteristics of two strategies of learning used in undergraduate studies in the health sciences: the Problem-Based Learning (PBL) and the Problem-solving Methodology (PSM). These methodological proposals are based on different theoretical conceptions and use problems as starting point for education and learning of students. In the PSM, the problems are formulated by the students from the comment of the reality. Already in the PBL, the problems are elaborated by a team of specialists to contemplate the knowledge and to reach planned learning objectives. With differentiated approaches and assumptions as to the meaning and objectives of the problematization process, the two proposals embody implications for teaching practice and the training of health practioners.
\end{abstract}

Key-words: Problem Based Learning, problem-solving, epistemology, health undergraduate.

\footnotetext{
${ }^{ \pm}$Uma parte deste trabalho foi apresentada na forma de painel no V Encontro Nacional de Pesquisa em Educação em Ciências (V ENPEC), Bauru, SP, dezembro de 2005.
} 


\section{INTRODUÇÃO}

"Ninguém educa ninguém, ninguém educa a si mesmo, os homens se educam entre si, mediatizados pelo mundo." (FREIRE, 2004. p.68).

No Brasil temos observado na última década a consolidação de propostas pedagógicas referentes ao ensino superior na área da saúde que assumem a necessidade da formação de profissionais críticos e reflexivos. O discurso oficial incorpora esta visão e preconiza a reorientação dos projetos pedagógicos dos cursos da área de modo a atender as demandas do Sistema Único de Saúde (BRASIL, 2006). Para tanto, segundo aquele discurso, há o imperativo em formar profissionais com capacidade para a compreensão e resolução de problemas que constituem a diversidade do cuidado e promoção da saúde do individuo e da sociedade (BRASIL, 2007).

O aparente consenso em torno daquela reorientação parece consolidar-se pela percepção social da crise por que passa a universidade brasileira (BUARQUE, 1994 p. 113) e pelo movimento que, como um desdobramento do processo de consolidação do Sistema Único de Saúde, tem reinvidicado mudanças na formação de profissionais da saúde (CECCIM e FEUERWERKER, 2004). Tal percepção tem suas raízes em fatores políticos e socioeconômicos complexos, bem como pela heterogeneidade social, étnica e cultural que historicamente marcou a formação da sociedade brasileira. Este contexto se reflete no interior das IES constituindo espaços coletivos de interlocução e enfrentamento com distintas concepções sobre a ciência e a natureza do conhecimento; manifestando-se na dinâmica característica da formação científica universitária, pautada pela persuasão, coptação, apropriação e (re)elaboração de discursos ideológicos, de visões epistêmicas e de conhecimentos e práticas ${ }^{1}$, os quais irão implicar no modelo de ensino, na estrutura das matrizes curriculares e nas práticas docentes hegemônicas.

Dentre as propostas de mudança tem se destacado a adoção de metodologias de aprendizagem ditas "ativas" que pronunciam a centralidade do processo no educando (ou “aprendiz") e assumem o discurso por um ensino problematizador. Sua estruturação e planejamento curricular têm como base a organização modular de conteúdos disciplinares e a adoção de estratégias de ensino-aprendizagem como a Aprendizagem Baseada em Problemas (ABP) (Problem Based Learning - PBL). Os defensores desta

\footnotetext{
${ }^{1}$ A dinâmica a que nos referimos faz referencia à transposição para o ambiente universitário de uma concepção socioconstrutivista da ciência, proposto pelo médico e epistemólogo Ludwik Fleck para explicar e compreender a gênese dos processos de produção e reprodução do discurso e do conhecimento científico. A este respeito ver: FLECK, L. La génesis y el desarrollo de un hecho científico. Madrid: Alianza Editorial, 1986.
} 
perspectiva têm justificado sua aplicação pela necessidade de aproximar teoria e prática, integrar conteúdos demasiadamente compartimentalizados, formar profissionais reflexivos, desenvolvendo sua capacidade para resolver problemas complexos, como aqueles que fazem parte do manejo clínico de pacientes (SCHMIDT, 1983).

As bases teóricas da $\mathrm{ABP}$, do ponto de vista epistemológico, bem como a identificação das teorias educacionais que lhe servem de suporte constituem o objeto de análise neste ensaio. Nesta perspectiva, ao discutir a ABP fazemos sua comparação com outra proposta de aprendizagem problematizadora que tem encontrado aceitação nos cursos da área da saúde: a Metodologia da Problematização, conforme apresentada e discutida por Berbel (1999).

Pretende-se abordar as características das duas metodologias, recorrendo a uma interpretação da concepção de educação problematizadora proposta por Paulo Freire, assim como a categoria de obstáculo epistemológico desenvolvida por G. Bachelard (1996) como contraponto à discussão. O maior desafio deste ensaio corresponde à sua pretensão de constituir um ponto de partida para a discussão sobre os limites, possibilidades e implicações de metodologias que, em sintonia com o discurso corrente entre os educadores e reformadores do ensino superior, se propõem problematizadoras.

\section{Aprendizagem Baseada em Problemas: Características e interfaces com TEORIAS DE APRENDIZAGEM}

A Aprendizagem Baseada em Problema (ABP), com o enfoque que hoje o conhecemos foi implantada como estratégia de ensino no final da década de 60 , na Universidade de McMaster, Canadá, e, pouco depois, na Universidade de Maastricht, Holanda (CAMP 2004). Desde então, várias universidades ao redor do mundo, têm adotado essa metodologia, inicialmente nos cursos da área da saúde, mas também na formação de áreas diversificadas como os cursos engenharias, economia, psicologia, arquitetura, física, química e biologia, entre outros.

No Brasil as instituições pioneiras na implantação da ABP foram a Faculdade de Medicina de Marília, o Curso de Medicina da Universidade Estadual de Londrina e a Escola de Saúde Pública do Ceará. O processo de aceitação desta metodologia poderia ser interpretado como uma reação ao modelo flexineriano de ensino na área da saúde (SANTOS, 1986), ainda hegemônico, que privilegia o ambiente hospitalar como espaço quase exclusivo para a aprendizagem de uma prática onde predomina o viés 
biologicista, característico do método clínico $^{2}$ (CYRINO e TORALLES-PEREIRA, 2004).

A ABP pode ser compreendida como uma estratégia ou metodologia de ensinoaprendizagem que objetiva a aquisição de conhecimentos no contexto de problemas clínicos (NORMAN, 1988).

De uma forma geral, o método tem por objetivo (a) desenvolver o raciocínio clínico e a capacidades do aluno em resolver problemas, (b) melhorar a aquisição, a retenção e o uso do conhecimento, (c) aproximar os conteúdos das ciências básicas e clínicas, (d) estimular o aprendizado autodirigido por parte do aluno, (e) estimular seu interesse pelo assunto ou conteúdo abordado e $(f)$ estimular estratégias mais eficazes de aprendizado desses conteúdos (BORDAGE, 1994 e SCHMIDT, 1993).

Essa metodologia parte do pressuposto da centralidade e da autonomia do aluno no processo de aprendizagem e apresenta como principais características a integração de conteúdos disciplinares estruturados sempre no contexto de um problema orientado para a discussão e resolução em grupo.

Constituindo as bases teóricas da ABP, os trabalhos de investigação na área da psicologia cognitiva, da aprendizagem do adulto e da neurociência ressaltam a importância da experiência prévia e da participação ativa na mobilização do aluno e como facilitador no processo de aquisição de conhecimentos. Por outro aquela metodologia tem na Teoria do Processamento da Informação sua principal base teóricometodológica (OLIVEIRA-FILHO, 2003).

Oliveira-Filho (2003) argumenta que, segundo esta teoria, três princípios orientam a aquisição de novas informações:

(a) ativação de conhecimentos prévios sobre o assunto;

(b) especificidade de codificação, implica que a posterior recuperação da informação é facilitada quando exemplos estão codificados juntamente com a informação, ou seja, quanto mais próxima a semelhança entre uma situação real e a situação de aprendizado, mais fácil a recuperação de informação, por este motivo os problemas elaborados devem se aproximar de situações reais do cotidiano do aluno, o que possibilita que sejam efetivamente significativos, condição imprescindível para o processo de problematização, e

\footnotetext{
${ }^{2}$ Sobre o método clínico ver FOUCAULT, M. O nascimento da clínica. $5^{\mathrm{a}}$. Ed., Forense Universitária, Rio de Janeiro (2003).
} 
(c) elaboração do conhecimento, seja através de resposta a perguntas elaboradas, ou de um contexto de interação em grupo onde o aluno verbaliza o seu conhecimento como aprende a partir da explicação dos colegas.

Estes princípios encontram-se contemplados na seqüência dos sete passos metodológicos propostos para a discussão e resolução do problema pelo grupo tutorial na ABP, conforme sugerido por Schmidt (1983):

(a) Leitura do problema, identificação e esclarecimento de termos desconhecidos;

(b) Identificação dos problemas propostos pelo enunciado;

(c) Formulação de hipóteses explicativas para os problemas identificados no passo anterior (nesta etapa os alunos utilizam seus conhecimentos prévios, aqueles que dispõem para a abordagem do problema);

(d) Resumo das hipóteses;

(e) Formulação dos objetivos de aprendizagem (de forma coletiva os alunos identificam e listam os assuntos que devem ser estudados para aprofundar os conhecimentos incompletos formulados nas hipóteses explicativas e que podem auxiliar na resolução do problema);

(f) Estudo individual dos assuntos levantados nos objetivos de aprendizagem;

(g) Retorno ao grupo tutorial para rediscussão do problema e compartilhamento no grupo dos novos conhecimentos adquiridos na fase de estudo anterior (nesta fase o grupo deve então apresentar uma síntese da sua discussão, elaborar e propor a resolução do problema).

Tootell e McGeorge (1998) ao discutir a contribuição de vários autores na fundamentação e desenvolvimento da $\mathrm{ABP}$, identificaram várias influências, que vão desde Bruner e Dewey, passando por Piaget, Kolb e Vygotsky. Kolb propõe um modelo teórico que denominou de aprendizagem vivencial, freqüentemente citado como referencial teórico para a ABP. Segundo este modelo a aprendizagem individual pode ser caracterizada em quatro momentos cíclicos: a) vivência (ou experiência) concreta, b) observação reflexiva, c) formação de conceitos abstratos e d) experimentação em novas situações. Este processo, representado pelo ciclo de Kolb, comporta duas dimensões: apreensão-conceituação e ação-reflexão (KOLB, 1990). A influência de distintos modelos teóricos que buscam explicar a aprendizagem também é discutida por Hughes Caplow e colaboradores (1997), para quem a ABP apresenta identidade com 
três enfoques teóricos: 1) a teoria do processamento da informação, 2) a perspectiva construtivista e, 3) a perspectiva da aprendizagem cooperativa.

Maudsley (1999) e Savery (2006) descrevem as diferenças na utilização da ABP como resultado da diversidade de concepções sobre o método. No entanto, a nosso ver, dependendo do pressuposto epistemológico e da concepção de educação do educador que aplica este método, haverá sentidos diferentes para o que significa "problematizar" no processo de aprendizagem, interferindo diretamente na consecução dos objetivos ao qual se propõe.

\section{A Metodologia da Problematização: algumas características}

Berbel (1999) cita o Método do Arco, de Charles Maguerez, como um dos primeiros referenciais teóricos que auxiliaram na fundamentação do que a autora irá desenvolver e denominar de Metodologia da Problematização (MP). Aquele Método, conforme apresentado por Bordenave e Pereira (1998), se desenvolve a partir da exposição do aluno à realidade ou a um recorte da mesma através de cinco etapas:

1) Observação da Realidade, etapa na qual os alunos são colocados frente a um problema inserido na realidade física ou social, possibilita uma visão global e contextualizada do problema;

2) A Segunda etapa é denominada Construção de uma maquete: corresponde à identificação das variáveis ou pontos-chave do problema, permite estabelecer os elementos estruturais e as relações entre eles na explicação e resolução do problema;

3) Na etapa de Teorização (discussão sobre a maquete) busca-se realizar a explicação, a fundamentação teórica do problema, seus elementos e relações, para isto os alunos são orientados a buscar subsídios na pesquisa bibliográfica, na consulta a bases de dados, leitura de livros, é o momento de identificar "a contribuição que as ciências podem dar ao esclarecimento do assunto" (BORDENAVE e PEREIRA, 1998). Esta fase da metodologia corresponde ao momento oportuno para a superação de obstáculos epistemológicos, onde os alunos podem efetivamente realizar o que Bachelard denomina catarse intelectual (BACHELARD, 1996); seguindo esta perspectiva o professor pode desempenhar um papel imprescindível na mediação do processo, através da investigação e identificação dos obstáculos (os seus e de seus alunos), orientando de forma mais adequada a etapa de teorização; 
4) Hipóteses de Solução, onde após ter realizado a fundamentação teórica, o estudo propriamente dito dos conteúdos deverá fornecer elementos para que os alunos, de forma crítica e criativa, proponham as possíveis soluções do problema.

5) Aplicação à Realidade (prática), esta etapa constitui um momento com potencial efetivamente transformador, na medida em que o aluno tem a possibilidade de superar o nível da formulação teórica, planejando estratégias que permitam, por em prática de alguma forma e em algum nível as sugestões elaboradas como solução do problema, estas sugestões deverão ser, de alguma forma, postas em prática ou encaminhadas. É um momento com forte presença do componente social e político, e conseqüentemente conscientizador e transformador da realidade. Uma forma de estabelecer e reforçar vínculos dos alunos com o seu meio.

Sobre a Metodologia da problematização e sua relação com o Arco de Marguerez, a autora afirma:

O Arco tem como ponto de partida a realidade vivida, aquela parcela da realidade onde o tema que está sendo ou será trabalhado está acontecendo na vida real (...). Ao desenvolver trabalhos com esta metodologia, os alunos ou os participantes são levados a observar a realidade de uma maneira atenta e irão identificar aquilo que na realidade está se mostrando como carente, inconsistente, preocupante, necessário, enfim problemático (BERBEL, 1999).

Enquanto metodologia de ensino-aprendizagem a MP encontra-se identificada, através do próprio testemunho de seus formuladores, com uma concepção históricocrítica de educação; segundo Vasconcellos (1999) ela vai encontrar seus pressupostos filosóficos, na fenomenologia, no existencialismo e em referenciais marxistas. Estes pressupostos partem

(...) da crença de que o homem se constrói e que pode ser sujeito quando integrado em seu contexto, reflete sobre ele e com ele se compromete (...) a concepção de aluno e de professor como sujeitos concretos, situados no tempo e no espaço, inseridos num contexto histórico (VASCONCELOS, 1999).

Do ponto de vista pedagógico a MP recebe a influência das vertentes críticas do construtivismo.

\section{COMPARANDO AS DUAS PROPOSTAS}

Alguns trabalhos têm procurado estabelecer comparações entre a ABP e a Metodologia da Problematização (CYRINO e TORALLES-PEREIRA, 2004; BERBEL, 1998). Apesar de utilizarem problemas como ponto de partida para a aprendizagem, 
pode-se perceber algumas diferenças fundamentadas sobre tudo na base teórica dessas metodologias (BERBEL, 1998). A consciência destas influências implica aos professores que desejem trabalhar com esta metodologia a formulação de algumas "perguntas epistemológicas" subjacentes ao método, que não podem ser ignoradas pelos educadores, sob pena de redução do método a um mero instrumental, um jogo de técnicas e procedimentos instrucionais (O'GRADY, 2004).

Nesta perspectiva, reduzido a um procedimento metodológico a ABP poderá ser utilizada com grande variação de intenções e resultados. As diferenças na aplicação da ABP poderiam ser interpretadas como incongruências de posições epistemológicas, que divergem sobre a essência do conhecimento; ou seja, refletem a relação de determinação entre o sujeito cognoscente e o objeto, a forma de compreender e proceder a apropriação do conhecimento na execução do método (O'GRADY, 2004).

Os problemas constituem um dos pontos comuns das duas propostas. Neste sentido, na sua concepção e formulação, estão inseridos pressupostos epistemológicos. Na MP, os problemas são identificados pelos alunos, através da observação, da sua confrontação com a realidade, enquanto na $\mathrm{ABP}$, eles são elaborados por um grupo de especialistas e contemplam a expectativa de que os alunos alcancem objetivos de aprendizagem inseridos em um planejamento educacional (CYRINO e TORALLESPEREIRA, 2004).

Mesmo que na MP o problema seja elaborado a partir da realidade manifesta e significativa para os alunos, com o objetivo de explicitar suas características e contradições; isto não garante a dimensão transformadora do processo. Desta forma, uma questão importante para qualquer método "problematizador" diz respeito ao papel que o problema desempenha na articulação entre o ato gnosiológico e o ato educativo.

\section{PROBLEMATIZAR COMO E PRA QUE ? APROXIMAÇÕES COM O PENSAMENTO Pedagógico de Paulo Freire}

Tanto a ABP, quanto a MP, partem do pressuposto que o aluno "sempre sabe algo sobre alguma coisa", ou seja, que o mesmo traz consigo conhecimentos prévios que o permitem interpretar e realizar uma primeira aproximação do objeto do conhecimento, do problema proposto. No entanto, não basta que esta premissa esteja declarada no método utilizado.

Há posições que defendem como um dos principais objetivos de uma educação problematizadora "conduzir o aluno à realidade" (IMBERÓN apud FRANCO, 2007). 
Esta perspectiva manifesta uma concepção de como e porque problematizar. A qual realidade se refere aquele discurso? Estaria o aluno solto no espaço? Qual o entorno no qual se desenvolve o processo de aprendizagem do aluno? Que compreensões o aluno já possui deste entorno? Que influências teriam na sua aprendizagem? O que dessas compreensões o professor conhece? Em outras palavras, caberia ao professor (re)conhecer o aluno como sujeito do conhecimento e o contexto de suas interações sociais (DELIZOICOV et al., 2002).

As posições defendidas por Paulo Freire (2004) instigam os educadores para o vivenciamento de uma postura fundamentada no compromisso em ajudar as pessoas a desenvolver seu potencial humano e não simplesmente treiná-las para a adaptação na sociedade. A propósito, encontra-se presente no senso comum pedagógico uma visão que algumas vezes se manifesta com expressões do tipo "é necessário ensinar aquilo que os alunos realmente precisam para a vida", que guarda contradições e significados distintos, os quais na crítica de Hamilton (2002) prioriza mais os processos e habilidades aos conhecimentos. É necessário questionar pra que serve esse aprendizado, não na perspectiva da acomodação ou enquadramento do indivíduo, mas ao contrário, problematizar só faz sentido quando inserido numa proposta de emancipação do ser humano.

Freire (2004) destaca na sua proposição pedagógica duas dimensões muito importantes: a dimensão problematizadora e a dimensão dialógica. Problematizar sob este olhar corresponde a uma dinâmica de articulação entre essas dimensões no processo de ensino-aprendizagem. Elas se posicionam como pontos de interface entre o conhecimento prévio do aluno e o conhecimento científico do professor, portador também das suas idiossincrasias, que auxiliam na delimitação do objeto do conhecimento, assim como na sua transformação no objeto de estudo sobre o qual se realizará a apropriação e (re)construção do conhecimento. Trata-se, de uma prática docente cujo eixo estruturador é a problematização, entendida em duas dimensões: 1 como busca de situações que envolvem a necessidade da emancipação do ser humano, as quais se tornariam os problemas a serem abordados no processo formativo, com a conseqüente contribuição que podem dar os conhecimentos da área de saúde; 2 - como procedimento mediador do diálogo entre conhecimento prévio do aluno e o conhecimento científico do professor em torno das situações eleitas como problemas (DELIZOICOV, 2001 e 2008). 
Para o professor, a dimensão problematizadora começa pela identificação das condições materiais e relações sociais significativas que determinam gêneses distintas dos conhecimentos que serão dialogados e problematizados no processo através da investigação temática e nas etapas sucessivas de codificação-problematizaçãodescodificação (FREIRE, 2004).

Intrínseco ao caráter dialógico-problematizador encontra-se o esforço e a possibilidade de compreensão e superação das situações e contradições que fazem parte do objeto de estudo. Como consequiência, sabem mais os alunos, que se apropriaram de novos conhecimentos e sabem mais os professores: mais sobre o próprio objeto de estudo, mais sobre o que ele significava e passou a significar para os alunos, mais sobre como ensinar.

\section{IMPLICAÇÕES PARA O ENSINO DE PROFISSIONAIS DA SAÚDE}

$\mathrm{O}$ modelo que imprimiu um tipo de racionalidade científica às práticas de intervenção em saúde, sem dúvida trouxe muitos benefícios ao cuidado das pessoas; no entanto operou também um deslocamento epistemológico do sujeito que padece de uma enfermidade para um conjunto de estruturas e processos biológicos alterados. Através da objetivação do indivíduo ocorreu o afastamento entre pacientes e profissionais cuidadores, com a deterioração da relação terapêutica, reduzindo a autonomia dos pacientes enquanto sujeitos no processo saúde-doença (SOARES e CAMARGO JUNIOR, 2007).

Neil Postman um crítico da excessiva submissão da sociedade moderna à tecnologia apresentou argumentações muito apropriadas para descrever a forma como a aceitação não-crítica do conhecimento científico pode sobrepujar valores, atacar liberdades, desfigurar o patrimônio cultural e acabar por impor um tipo de dominação que o autor denomina Tecnopólio (POSTMAN, 1994).

$\mathrm{Na}$ área da saúde a utilização excessiva (e por vezes desnecessária) da tecnologia nas intervenções e cuidados ao paciente faz parte de um fenômeno característico denominado medicalização social (ILLICH, 1975). Considerando que o uso dessa tecnologia tem um custo e está associado ao risco nos indicadores de morbidade e da mortalidade relacionada ao impacto da sua utilização; torna-se evidente a necessidade de desenvolver, durante a formação dos profissionais da saúde, competência científica para a apropriação crítica desse tipo de tecnologia. A questão que se impõe diz respeito a como mediar esta apropriação, no contexto das diferenças 
entre os alunos e das situações de aprendizagem programadas em currículos que, apesar do discurso, ainda priorizam um processo fragmentado característico do ensino tradicional. É nesta perspectiva que dois princípios propostos por Paulo Freire têm um grande significado para uma prática de ensino problematizadora: o compromisso por uma educação libertadora, no sentido do desenvolvimento da autonomia do indivíduo; e o princípio da dialogicidade como exercício pedagógico constantemente em construção daquela prática (FREIRE, 2004).

Podemos considerar que na proposta da MP há uma manifesta opção por este caminho. No entanto há a possibilidade de instrumentalizar a ABP com este enfoque; o que implica em uma outra intencionalidade do processo formativo e a necessidade de ser voluntariamente incorporada pelo educador quando da implantação e utilização da $\mathrm{ABP}$.

Freire argumenta que o ato de formar, educar o cidadão, consiste num processo de "ensinar a pensar certo"; ou seja, significa ir além da transmissão de conteúdos, instigando o educando a exercer a reflexão crítica e transformadora da realidade, onde este educando trabalha a partir da sua própria realidade. Este processo é apresentado como uma atividade que deve ser compartilhada com os educandos, pois "não há docência sem discência", visto sob está ótica, ensinar é uma atividade que não existe sem o ato de aprender (FREIRE, 1996).

Quanto mais se problematizam os educandos, como seres no mundo e com o mundo, tanto mais se sentirão desafiados (FREIRE, 2004).

Encontramos na MP uma identificação com esta visão de mediação, historicamente determinada e não-neutra do ensino e da aprendizagem como um processo de enfrentamento da realidade pelo homem.

A educação como prática de liberdade, ao contrário daquela que é prática da dominação, implica a negação do homem abstrato, isolado, solto, desligado do mundo, assim como também a negação do mundo como uma realidade ausente dos homens (FREIRE, 2004).

Estas aproximações teóricas, quando consideramos que o indivíduo interage com o objeto do conhecimento, com os fenômenos que constituem a realidade, mediado pelas relações sociais, nos ajudam a compreender a importância das concepções prévias, do senso comum dos alunos na abordagem de ensino dos problemas, na apropriação do conhecimento, mas também na constituição dos obstáculos epistemológicos a serem superados no processo de problematização. Neste sentido, em função da explicitação da concepção de sujeito apresentada pelos formuladores da MP está mais próxima de uma 
perspectiva problematizadora como sugerido no pensamento de Paulo Freire, ainda que a esta característica não se deva associar um caráter de superioridade quando cotejada com a ABP. No entanto, a ABP em função das múltiplas contribuições teóricas que fundamentam sua base conceitual, comporta internamente contradições que podem constituir obstáculos ao processo de aprendizagem.

\section{IMPLICAÇÕES ÉTICAS PARA O ENSINO}

Uma das contribuições significativas da formação universitária na área da saúde corresponde à construção de uma cultura científica e tecnológica. Jamais a humanidade esteve diante de tantos desafios e dilemas mediados ou influenciados diretamente por um nível de desenvolvimento científico e tecnológico como na atualidade. No campo da saúde, como uma conseqüência negativa deste desenvolvimento vimos o intenso processo de medicalização da sociedade e da vida. Uma proposta de ensino problematizadora consiste em fomentar nos estudantes a apropriação crítica do conhecimento científico, considerando que estes, como profissionais ou mesmo como cidadãos, têm seu modo de vida, suas relações sociais e no mundo do trabalho, influenciados pelos resultados da ciência e da tecnologia contemporânea.

Na perspectiva de Bachelard (1996), o professor reconhece nos conhecimentos prévios dos alunos, obstáculos epistemológicos e promove, por meio de rupturas do conhecimento vulgar, a possibilidade de o aluno realizar a catarse intelectual e afetiva desse conhecimento para que o mesmo possa romper com o conhecimento do senso comum e transitar também pelo conhecimento científico. Em relação a este último, é necessário que o mesmo esteja exposto constantemente ao confronto, à dialetização.

No entanto é preciso ter claro que identificar e reconhecer o obstáculo epistemológico dos alunos não é suficiente. Faz-se necessário ao professor identificar também os obstáculos pedagógicos inerentes entre outros, às práticas e metodologias pedagógicas utilizadas em sala de aula. Trabalhar com os alunos a problematização do conhecimento pode ser uma estratégia que possibilita a superação desses obstáculos, neste sentido vemos tanto a ABP como a MP como metodologias interessantes e aplicáveis, não só para o ensino na área da saúde, ou para o ensino superior. Parece claro que a questão não é apenas problematizar, mas o quê ? e porquê ? problematizar.

Em último caso, mais do que formar "profissionais competentes", corresponde ao compromisso intelectual e político do professor aliar à formação profissional e 
científica dos alunos valores como o sentido ético de preocupação e solidariedade com o outro; de inclusão e aceitação das diferenças (mas de rejeição absoluta das desigualdes e iniqüidades sociais), de preservação da vida e do mundo natural.

Compreender a Problematização como um processo de "reflexão sobre os homens em suas relações com o mundo" (FREIRE, 2004), onde o conhecimento se constrói pelo diálogo constante entre o sujeito-educando-educador e sujeito-educadoreducando, tendo como objeto desse diálogo sua interação com a realidade, constitui um passo fundamental para uma metodologia que se pretenda problematizadora; sob o risco de a própria metodologia representar mais um obstáculo para a aprendizagem, do que um instrumento de libertação.

\section{Referências bibliográficas}

BACHELARD, G. A formação do espírito científico: contribuição para uma psicanálise do conhecimento. $1^{\mathrm{a}}$ ed. Rio de Janeiro: Contraponto, 1996.

BERBEL, N. A. N. "A problematização e a aprendizagem baseada em problemas: diferentes termos ou diferentes caminhos?" Interface - Comunic., Saúde, Educ., v. 2, p. $139-154,1998$.

BERBEL, N. A. N. A metodologia da problematização e os ensinamentos de Paulo Freire: uma relação mais que perfeita. In: BERBEL, N. A. N. (org.). Metodologia da Problematização: fundamentos e aplicações. Londrina: Editora UEL, 1999. pp. 1-27

BORDAGE, G. "Elaborated knowledge: a key to successful diagnostic thinking". Academic Med., v. 69, p. 883 - 885, 1994.

BORDENAVE, J. D.; PEREIRA, A. M. Estratégias de ensino-aprendizagem. 19ª ed., Petrópolis: Editora Vozes, 1998.

BRASIL. Ministério da Saúde. Ministério da Educação. Programa Nacional de Reorientação da Formação Profissional em Saúde - Pró-Saúde: objetivos, implementação e desenvolvimento potencial/Ministério da Saúde, Ministério da Educação. - Brasília : Ministério da Saúde, 2007. 86 p.

BRASIL. Ministério da Educação. Instituto de Estudos e Pesquisas Educacionais Anísio Teixeira (INEP). A trajetória dos cursos de graduação na área da saúde: 1991 - 2004. Brasília: Instituto de Estudos e Pesquisas Educacionais Anísio Teixeira, 2006. 15 v.

BUARQUE, C. A aventura da universidade. São Paulo: Editora da UNESP; Rio de Janeiro: Paz e Terra, 1994, 239p.

CAMP, G. "Problem-based learning: A paradigm shift or a passing fad?" Medical Education Online, v. 1, n. 2, 1996. Disponível em:

http://www.utmb.edu/meo/f0000003.htm. Acesso em: 21/07/2004.

CECCIM, R. B.; FEUERWERKER, L. C. M. Mudança na graduação das profissões de saúde sob o eixo da integralidade. Cad. Saúde Pública, v. 20, n. 5, p. 1400 - 1410, 2004. 
CYRINO, E. G.; TORALLES-PEREIRA, M. L. Trabalhando com estratégias de ensino-aprendizado por descoberta na área da saúde: a problematização e a aprendizagem baseada em problemas. Cad. Saúde Pública, v. 20, n. 3, p. 780-788, 2004.

DELIZOICOV, D. La Educación en Ciencias y la Perspectiva de Paulo Freire. Alexandria: Revista de Educação em Ciência e Tecnologia, v.1, n.2, p.37 - 62, 2008.

DELIZOICOV, D. Problemas e Problematizações. In: PIETRECOLA, M. (org.). Ensino de Física:conteúdo, metodologia e epistemologia numa concepção integradora. Florianópolis: Ed. UFSC, 2001.

DELIZOICOV, D.; ANGOTTI, J. A.; PERNAMBUCO, M. M. Ensino de ciências: fundamentos e métodos. São Paulo: Cortez, 2002.

FLECK, L. La génesis y el desarrollo de un hecho científico. Madrid: Alianza Editorial, 1986.

FRANCO, A. C.; BOOG, M. C. F. Relação teoria-prática no ensino de educação nutricional. Rev. Nutr., v. 20, n. 6, 2007. Disponível em: $<\mathrm{http}: / / \mathrm{www}$.scielo.br/scielo.php?script=sci_arttext\&pid=S141552732007000600007\&lng=en\&nrm=iso>. Acesso em: 08/09/2008.

FREIRE, P. Pedagogia do Oprimido. 38a. ed., Rio de Janeiro: Paz e Terra, 2004.

FREIRE, P. Pedagogia da autonomia: Saberes necessários à prática educativa. São Paulo: Paz e Terra, 1996. 165 p.

HAMILTON, D. O revivescimento da aprendizagem? Educação \& Sociedade, v. 23, n. 78, p. $187-198,2002$.

HUGHES CAPLOW, J. A.; DONALDSON, J. F.; KARDASH, C.; HOSOKAWA, M. Learning in a problem-based medical curriculum:students' conceptions. Medical Educ., v. 31, p. $440-447,1997$.

ILLICH, I. A expropriação da saúde: nêmesis da medicina. $2^{\mathrm{a}}$. ed. Rio de Janeiro: Nova Fronteira, 1975.

KOLB, D. Psicologia organizacional: uma abordagem vivencial. São Paulo: Atlas, 1990.

MAUDSLEY, G. Do we all mean the same thing by "problem-based learning"? A review of the concepts and a formulation of the ground rules. Academic Medicine, v. 74, n. 2, p. $178-85,1999$.

NORMAN, G. R. Problem-solving skills, solving problems and problem-based learning. Medical Educ., v. 22, p. 279 - 286, 1988.

O'GRADY, G. The Dangers of PBL (and other instructional fads): Beware the epistemological hole in the practice of PBL". 5th Asia-Pacific Conference on PBL, Mar. 2004 and PBL 2004 Conference, Cancun, Mexico, Jun. 2004. Disponível em: http://discovery.rp.edu.sg/home/CED/research/papers/dangers_of_pbl2.pdf. Acesso em: 13/08/2004.

OLIVEIRA FILHO, GR. Bases Teóricas para a Implementação do Aprendizado Orientado por Problemas na Residência Médica em Anestesiologia. Rev. Bras. Anestesiol., v. 53, n. 2, p. $286-299,2003$. 
POSTMAN, N. Tecnopólio: a Rendição da Cultura à Tecnologia. São Paulo: Nobel, 1994.

SANTOS, J. O. Filosofia da educação médica: interpretação da práxis. Rev. Bras. Educ. Med. v. 10, n. 2, p. $82-6,1986$.

SAVERY, J. R. Overview of Problem-based Learning: Definitions and Distinctions. The Interdisciplinary Journal of Problem-based Learning, v. 1, n. 1, p. 9 - 20, 2006.

SCHMIDT, H. G. Foundations of problem-based learning: some explanatory notes. Medical Educ., v. 27, p. 422 - 432, 1993.

SCHMIDT, HG. Problem-based learning: rationale and description. Medical Education, v. 17 , p. $11-16,1983$.

SOARES, J. C. R. S.; CAMARGO JUNIOR, K. R. Patient autonomy in the therapeutic process as a value for health. Interface - Comunic., Saúde, Educ., v. 11, n. 21, p. 65 78, 2007.

TOOTELL, K.; MCGEORGE, D. An investigation of the use of problem based learning in Professional Degrees. Australian Association for Research in Education (AARE) Conference, Adelaide, nov. de 1998. Disponível em: http://www.aare.edu.au/98pap/too98077.htm. Acesso em: 21/07/2004.

VASCONCELLOS, M. M. M. Aspectos pedagógicos e filosóficos da Metodologia da Problematização", In: Berbel, N. A. N. (org.). Metodologia da Problematização:

fundamentos e aplicações. Londrina: Editora UEL, 1999. p.29-59 